\title{
COGNITIVE SCALE-FREE NETWORKS AS A MODEL FOR INTERMITTENCY IN HUMAN NATURAL LANGUAGE
}

\author{
PAOLO ALLEGRINI \\ ILC-CNR Area della Ricerca di Pisa, via Moruzzi 1, 56010 Pisa, Italy \\ E-mail: allegrip@ilc.cnr.it \\ PAOLO GRIGOLINI \\ Dipartimento di Fisica, Università di Pisa and INFM, via Buonarroti 2, 56127 Pisa Italy \\ Center for Nonlinear Science, UNT, P.O. Box 311427, Denton, Texas 76203-1427 \\ IPCF-CNR, Area della Ricerca di Pisa, via Moruzzi 1, 56010 Pisa, Italy \\ E-mail: grigo@df.unipi.it \\ LUIGI PALATELLA \\ Dipartimento di Fisica, Università di Pisa and INFM, via Buonarroti 2, 56127 Pisa Italy \\ E-mail: grigo@df.unipi.it
}

\begin{abstract}
We model certain features of human language complexity by means of advanced concepts borrowed from statistical mechanics. Using a time series approach, the diffusion entropy method (DE), we compute the complexity of an Italian corpus of newspapers and magazines. We find that the anomalous scaling index is compatible with a simple dynamical model, a random walk on a complex scale-free network, which is linguistically related to Saussurre's paradigms. The model yields the famous Zipf's law in terms of the generalized central limit theorem.
\end{abstract}

\section{Introduction}

Semiotics studies linguistic signs, their meanings, and identifies the relations between signs and meanings, and among signs. The relations among signs (letters, words), are divided into two large groups, namely the syntagmatic and the paradigmatic, corresponding to what are called Saussurre's dimensions 1]. These dimensions are analogous to physical concepts like time and space. One can grasp an understanding of them by looking at Fig. 1 The abscissa axis represents the syntagmatic dimension, while the ordinate axis represents the paradigmatic one. Along the abscissa grammatical rules pose constraints on how words follow each other. This dimension is a temporal one, with a casual order. An article (as "a" or "the"), e.g., may be followed by an adjective or a noun, but not by a verb of finite form. At a larger "time-scale", pragmatic constraints rule the succession of concepts, to give logic to the discourse. The other axis, on the other hand, refers to a "mental" space. The speaker has in mind a repertoire of words, divided in many categories, which can be hierarchically complex and refer to syntactical or semantic "interchangeability". Different space-scales of word paradigms can be associated to different levels of this hierarchy. After an article, to follow the preceding example, one can choose, at a syntactical level, among all nouns of a dictionary. However, at a deeper level, semantic constraints reduce the available words to be chosen. For instance, after "a dog" one can choose any verb, but in practice only among verbs selected by semantic constraints (a dog runs or sits, but does not read or smoke). The sentence "a dog graduates", for instance, fits paradigmatic and syntagmatic 
rules behind Fig. 1, but the semantics would in general forbid the production of such a "nonsensical" sentence.

The two dimensions are therefore not quite orthogonal, and connect, e.g., at a cognitive level. The main focus of this paper is to show that this connection is in fact reproduced at all scales. We shall also show that both dimensions are scale free and that the complexity of linguistic structures in both dimensions can be taken into account in a unified model, which is able to explain most statistical features of human language, including, at the largest scales, the celebrated Zipf's law 2 ].

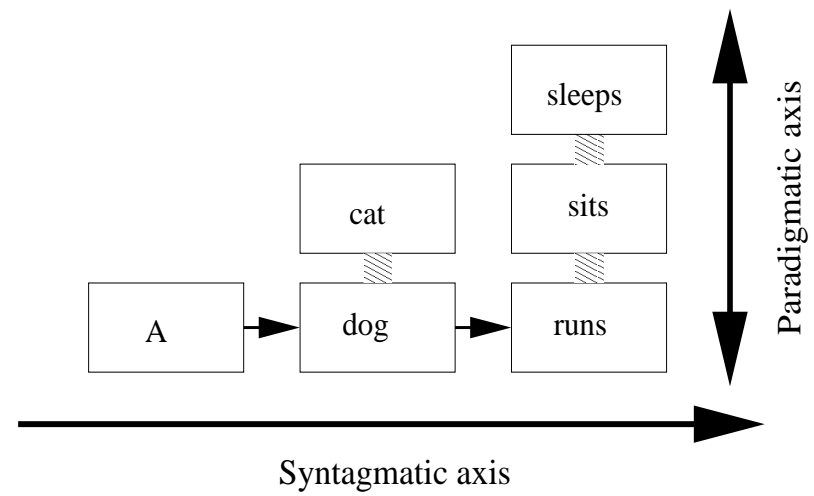

Figure 1. Saussurre's dimensions. In this example the first position in the syntagmatic axis is an article, the second a noun and the third a verb in the third person.

Zipf's law relates the rank $r$ of words to their frequency $f$ in a corpus. Remarkably, this does not mean that the probability of a word is actually defined. In fact, a word may have a small or large frequency depending on the genre of the corpus (i.e. a large collection of written text) under study, and even two extremely large corpora of the same type fail in reproducing the same word frequencies. It is however remarkable that the occurrence of words is such that for any corpus and for any natural language a property emerge so that one finds only few frequent words and a large number of words encountered once or twice. Let us define word rank $r$, a property depending on the corpus adopted, as follows. One assigns rank 1 to the most frequent word, rank 2 to the second frequent one, and so on. Each word is uniquely associated to a rank, and, although this number varies form corpus to corpus, one always finds that

$$
f \propto \frac{1}{r}
$$

This property means that word frequencies do not tend to well-defined probabilities. We assume that what can be defined is a "probability of having a frequency" $P(f)$ for a randomly selected word. Operatively, one measures $P(f)$ by counting how many words have a certain frequency $f$. In Section III we show that a $P(f)$ compatible with the Zipf's law can be derived from the model proposed herein, thus 
providing our model an experimental support. For our scopes, we assume a statistical mutual independence for the occurrence of different concepts. This hypothesis is appealing, since it means that every and each occurrence of a concept makes entropy increase, thus identifying the mathematical information (i.e. entropy) with the common-sense information (i.e. the occurrence of concepts). Unfortunately, a concept is not, a priori, a well defined quantity. Herein we assume that concepts are represented by words (or better by lemmata) or by groups of semantically similar words or lemmata ${ }^{a}$.

Because of the mutual independence among different concepts, we can extract from a single corpus as many "experiments" as the number of concepts. For each experiment we select only one concept and we mark the occurrence of the selected word or group of words corresponding to this concept. For the analysis we use the recently developed Diffusion Entropy (DE) method, which is able to identify whether a marker is a "real event", i.e. it carries maximal information, and to extract the scaling properties of the language dynamics. We show here that anomalous scaling (different from Brownian motion) is an indication of long-range correlations of the series, and that in fact these properties are well measured by the DE, even if the marker is not identified with absolute precision.

The overall dynamics, given by the flow of concepts over time, experimentally mirrors the dynamics of intermittent dynamical systems, like the Manneville's Map: These systems have long periods of quiescence followed by bursts of activity. This variability of waiting times between markers of activity is responsible for long-range correlations $\underline{3}$.

The second aspect of the paper is the connection between space and time complexity, and its application to linguistics. We will assume that atomic concepts exist and represent nodes of a complex network, connected by arcs representing, when existing, semantic associations between a concept and another. We assume that our markers are actually defined as a group of neighboring nodes. We then assume that language can be produced by a random walker, "associatively" traveling from concept to concept. The scale-free properties of the network, independently measured by our research group, provides a bridge to understand the intermittent dynamics earlier described. In this unified model the network is a representation of Saussurre's paradigms, whose complexity mirrors the syntagmatic one in the asymptotic limit.

\section{DE and concepts}

Let us review the DE method 451677. In synthesis, one defines a "marker" on a time sequences, and studies the probability $p(x ; t)$ of having a number $x$ of markers in a window of length $t$. This statistical analysis is done by moving a window of length $t$ along the sequences, counting how many times one finds $x$ markers inside this window, and dividing this number by the total number $N-t+1$ of windows of size $t$, where $N$ is the total length.

${ }^{a} \mathrm{~A}$ lemma is defined as a representative word of a class of words, having different morphological features. For instance the word "dogs" has lemma "dog", and word "sleeping" has lemma "sleep".

ALLEGRINI, GRIGOLINI, PALATELLA 
Having large number values for $x$ and $t$, we can adopt a continuous approximation. Moreover, in the ergodic and stationary condition, a scaling relation is expected, namely

$$
p(x ; t)=\frac{1}{t^{\delta}} F\left(\frac{x-w t}{t^{\delta}}\right),
$$

where $w$ is the overall marker density, $\delta$ is the scaling index and $F$ is a function. If $F$ is the Gauss function, $\delta$ is the known Hurst index, and if the further condition $\delta=0.5$ is obeyed, then the process is said to be Poissonian, and the dynamics of $x$ is called "Brownian motion". If this condition applies, there is no long-range memory regulating the occurrence of markers in time.

It is straightforward to show that $S(t)=\int_{-\infty}^{\infty} d x p(x ; t) \ln p(x ; t)$, namely the Shannon Information, with condition (2), leads to

$$
S(t)=k+\delta \ln t
$$

where $k$ is a constant. The evaluation of the slope according to which $S$ increases with $\ln t$ provides therefore a measure for the anomalous scaling $\delta$.

Let us briefly mention what we know about applying DE to time series with known long-range correlation. We construct an artificial series by letting $\xi_{i}=1$ (this means that we find the marker at the $i$ th position), or $\xi_{i}=0$ (the $i$-th sign is not a marker). We then assume "informativity" for the marker (markers are then called "events"), namely that the distance between a " 1 " and the successive does not depend on the such previous distances. Then, if the distances $t$ between events are distributed as

$$
\psi(t)=(\mu-1) \frac{T^{\mu-1}}{(t+T)^{\mu}}
$$

$\left(\psi(t) \sim t^{-\mu}\right.$ asymptotically is a sufficient condition), then the theory based on continuous-time random walk and on the generalized central-limit theorem yields for $p(x ; t)$ a truncated Lévy probability distribution function (PDF) 4 . DE detects the scaling $\delta$ of the central part, namely

$$
\delta=\frac{1}{\mu-1} \text { if } 2<\mu<3, \delta=0.5 \text { if } \mu>3 .
$$

The condition $2<\mu<3$ means long-range correlation, since for truncated Lévy PDFs asymptotically $\left\langle x^{2}(t)\right\rangle-\langle x(t)\rangle^{2} \propto t^{4-\mu}$ and therefore the correlation function decays as $t^{\mu-2}$. Note that the decay of this correlation function is non-integrable, yielding an infinite correlation time. The theory rests on a dichotomous $\xi$, and experimentally this means the presence or absence of a certain marker. One may, for instance look for a certain letter, so that the time is the ordinal number of the typographical characters in the text. As later shown, we have better results by looking at lemmata, where the "time" is the ordinal number of words. We shall show that, with a good choice of semantic markers, Eq. (4) is a good model for concepts dynamics in natural language.

ALLEGRINI, GRIGOLINI, PALATELLA 
Eq. (5) 4 rests on uncorrelated waiting times between events. This means that if two markers are separated by intervals of words of duration $\tau_{k}$ (the distance in words between the $k$-th and the $k+1$-th occurrence of the marker) then $\left\langle\tau_{i} \tau_{j}\right\rangle \propto \delta_{i j}$, where $\delta_{i j}$ is the Kroeneker delta. Under these conditions each event carries the same amount of information. The statistical independence between the $\tau_{k}$ intervals means that the information carried by the events is maximal for a given waiting time distribution $\psi(\tau)$. In a linguistic jargon, we can say that if in a corpus we find a marker (e.g. a list of words) such that $\delta \approx 1 /(\mu-1)$ then this marker is informative in that corpus. For didactical purposes, we shall see that certain markers, e.g. punctuation marks, are not real events, but are rather modeled by a Copying Mistake Map (CMM) 8 . This means that discourse complex dynamic is such that the punctuation marks actually carry long-range correlations, and anomalous scaling in the PDF, while the waiting times between such marks are correlated. Punctuation marks are not informative. Their complexity is just a projection of a complexity carried by "concept dynamics".

\subsection{The CMM and non-informative markers}

The Copying Mistake Map (CMM) 8 is a model originally introduced to study the anomalous statistics of nucleotides dispersion in coding and non-coding DNA regions. The CMM is a combination of two sequences: We have an "original" time sequence like e.g. the long-range-correlated series earlier discussed, corresponding to the waiting time distribution (4). Then, for any $\xi_{i}$ we either leave it unchanged with probability $\epsilon$ or change it with a completely random value with probability $1-\epsilon$ (copying mistake).

The resulting waiting time distribution decays exponentially, since the probability of finding a 1 after a time $t$ from the preceeding one, is given by two terms. This is because the 1 can be associated to two kinds of origin: it may be an "original" 1 , or an original "zero" flipped by the copying mistake. We can write the "experimental" waiting-time distribution $p s i_{e x p}(t)$, in terms of $p s i_{c o r r}(t)$ of the mentioned long-range-correlated model (44), and of $p s i_{\text {rand }}(t)$ of the Poissonian copying process, namely

$$
\psi_{\text {exp }}(t)=\psi_{\text {rand }}(t) \Psi_{\text {corr }}(t)+\Psi_{\text {rand }}(t) \psi_{\text {corr }}(t),
$$

where $\Psi(t) \equiv \int_{t}^{\infty} d t^{\prime} \psi\left(t^{\prime}\right)$ and $\Psi_{\text {rand }}(t) \equiv \int_{t}^{\infty} d t^{\prime} \psi_{\text {rand }}\left(t^{\prime}\right)$, and

$$
\psi_{\text {rand }}(t)=\ln \left(\frac{2}{1-\epsilon}\right) \cdot\left(\frac{2}{1-\epsilon}\right)^{-t} .
$$

Since $\psi_{\text {rand }}(t)$ and consequently $\Psi_{\text {rand }}(t)$ decay as an exponential function, so it does, in the asymptotic limit, $\psi_{\exp }(t)$. What about the DE curve? The theory predicts 9 a random $(\delta=0.5)$ behavior for short times, a knee, and a slow transition to the totally correlated behavior. An example of CMM is given by punctuation marks in Natural Language. We choose punctuation marks as markers for an Italian corpus of newspaper and magazines, of more than 300,000 words length, 
called Italian Treebank (hereafter TB). In this experiment we look at words, and we put a 0 for every word which is not a punctuation mark, and a 1 when we find such a mark (full stops, commas, etc.). A sentence like "Felix, the cat, sleeps!" is therefore transformed into "0 $\begin{array}{llllllll}0 & 0 & 1 & 0 & 1 \text { ". }\end{array}$

Fig. 2 shows that this markers lead to a time series with all the earlier exposed features of a CMM. This means that the waiting times $\tau_{k}$ are correlated, and therefore punctuation marks are not events. Notice however that an asymptotic anomalous $\delta$ is detected by $\mathrm{DE}$, and therefore there is a long-range correlation in the text, which may be carried by some other more informative marker.

\subsection{Concepts as informative markers}

More experiments, not reported here, show that the CMM behavior is typical for many characters, and are shared by all the letters of the alphabet, with a $\delta \approx 0.6$. Passing from a "phonetic" (in Italian we can assume that alphabetic characters mirror the phonetic) to a morpho-syntactic level is linguistically interesting. To do so, a text has to be lemmatized and tagged with respect to its part of speech. After this procedure we can identify as a marker the occurrence of a certain part of speech (e.g. article, adverb, adjective, verb, noun, preposition, numeral, punctuation etc.). For instance, the sentence "Felix, the cat, sleeps!" is now transformed into "N P R N P V P", where N, P, R and V stand for nouns, punctuation, article and verb. If we select the occurrence of verb as a marker, then we have "0 $\begin{array}{llllllll}0 & 0 & 0 & 0 & 1 & 0\end{array}$ ". Fig 3 shows the result of this experiment for verbs and for numerals. We notice that we have a similar behavior for the $\mathrm{DE}$, and a completely different behavior for the evaluation of the waiting-times distribution $\psi(t)$ (where $t$ is the number of words between markers). We notice that DE reveals a long-time correlation, while, $\psi(t)$ shows an exponential truncation at long times. However, in the case of numerals we find a large transient with a slope $\mu_{\text {numerals }}<2$, and therefore a non-stationary behavior. This is in fact due to the uneven distribution of numerals in the corpus, since they are encountered more often in the economic part of the Italian newspapers. However, this still unsatisfactory result for numerals reveals that this kind of markers is more informative than a phonetic one or than the presence of verbs. This is linguistically interesting, since numerals denote a part of speech, but also a "semantic class".

We are therefore led to suppose that informative markers are the ones associated with a semantically coherent class of words. This is however a problem, since every single concept is too rare in a balanced corpus (a long text with a variety of genres). The next level of our exploratory search for events is therefore to look at the occurrence of "salient words" in a specialistic text. Such a corpus has been made available as the Italian corpus relative to the European project POESIA 10. POESIA is a European Union funded project whose aim is to protect children from offensive web contents, like, e.g. pornography in WWW URLs. Salient "pornographic" words were automatically extracted by comparing their frequency in an offensive corpus, with respect to the balanced TB-corpus. The definition adopted

ALLEGRINI, GRIGOLINI, PALATELLA 

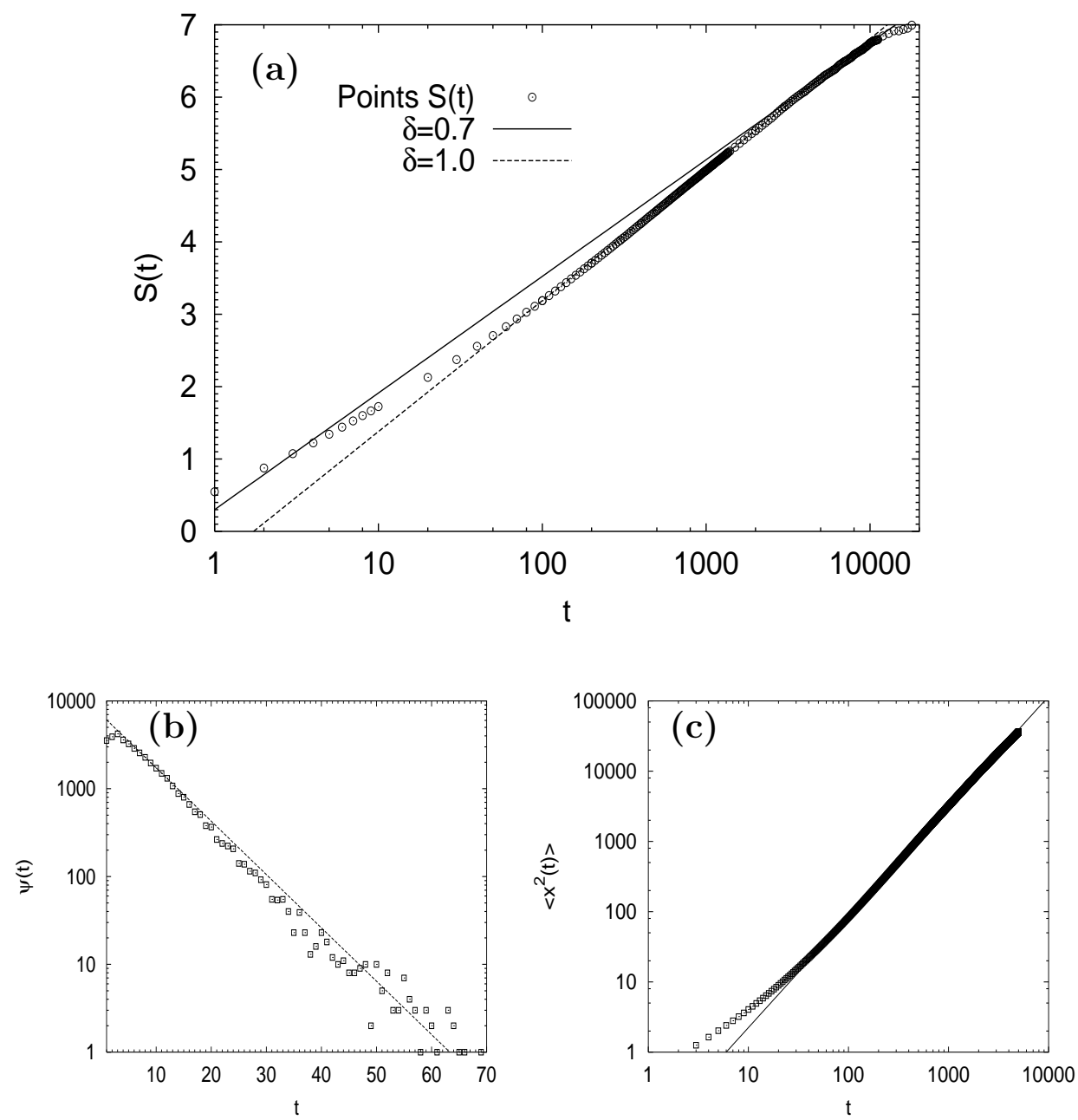

Figure 2. (a) Diffusion entropy for punctuation marks. The fit for the asymptotic limit (solid line) yields a $\delta=0.7$. The dashed line marks a transient regime with $\delta=1$. (b) Non-normalized distribution of waiting times for punctuation marks, namely counts of waiting times of length $t$ between marks in TB. The expression for the dashed line fit is $7000 \cdot \exp (-t / 7.15)$. (c) Second moment analysis for punctuation marks. The expression for the solid line fit is $0.06 \cdot t^{1.57}$. Notice that $1.57 \approx 3-1 / 0.7$, namely the expression $H=3-1 / \delta$ of Ref. 9 for Lévy processes stemming from CMM's is verified.

was

$$
s(l)=\frac{f_{E C}(l)-f_{T B}(l)}{f_{E C}(l)+f_{T B}(l)},
$$

where $f_{E C}(l)$ is the frequency, in the erotic corpus, of the lemma $l$, and $f_{T B}(l)$ is the same property in the reference Italian corpus (Italian Treebank). Salient 

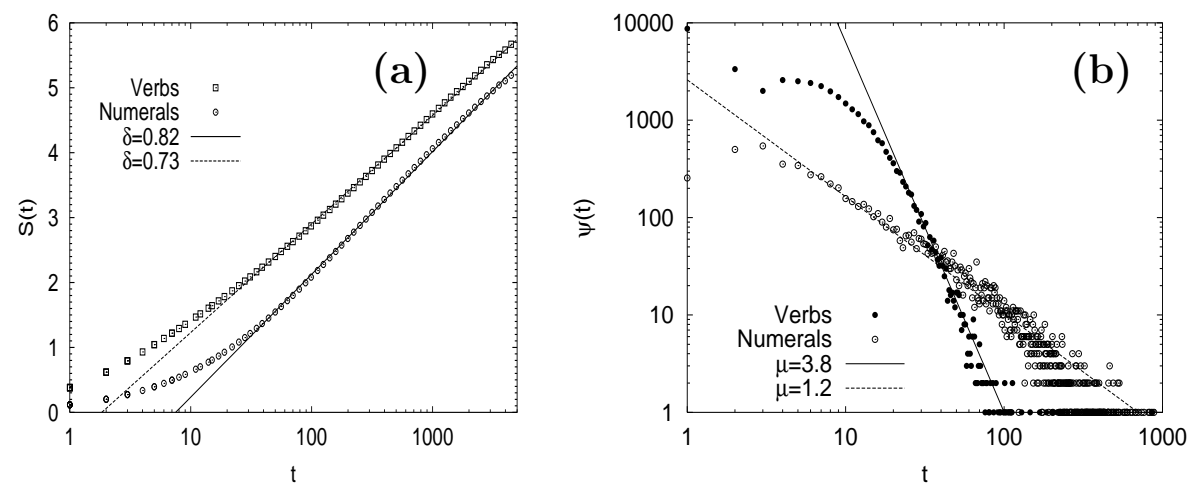

Figure 3. a) DE for verbs (squares) and numerals (circles). The dashed line is a fit for the verbs, with $\delta=0.73$, while the solid line is a fit for the numerals, with $\delta=0.82$. b) $\psi(t)$ for verbs (black circles) and numerals (white circles). The dashed line is a fit for the numerals, with $\mu=1.2$, while the solid line is a fit for the verbs, with $\mu=3.8$.

lemmata were automatically chosen as the $5 \%$ with the highest value of $s$. Notice that in this experiment all "dirty" words are not taken into consideration, because they do not appear in the reference corpus, and therefore $s$ cannot be properly defined. However an offensive metaphoric use of terms is in fact detected, leading to a completely new way to automatic text categorization and filter 10, using a method, based on DE analysis, called CASSANDRA 11 .

Salient words were therefore used as markers for our analysis, as earlier described. The results are shown in Fig. 4, clearly showing that in a specialized corpus, salient words of this genre, pass the test of informativeness. Salient words, and plausibly words in general, are therefore distributed like markers generated by an intermittent dynamical model, with $\mu \approx 2.1$ and, in agreement with (15), $\delta \approx 1 /(\mu-1)=0.91$. We see in the next section how this behavior is plausibly connected with a topological complexity at the paradigmatic level, and in Section III we derive the Zipf's law from the resulting model.

\section{Scale-free networks, intermittency and the Zipf's law}

In this section we build a cognitive model for connecting structure and dynamics. Allegrini et al. 12 identified semantic classes in the Italian corpus, by looking at paradigmatic properties of interchangeability of classes of verbs with respect to classes of nouns. They defined "superclasses" of verbs and nouns as "substitutability islands", namely groups of nouns and verbs sharing the properties that in the corpus you find each verb of the class co-occurring, in a context, with each noun of the class 12. This is precisely a direct application of the notion of "paradigm". Let us call $p_{v}(c)$ and $p_{n}(c)$, respectively, the number of verbs or nouns belonging to a 

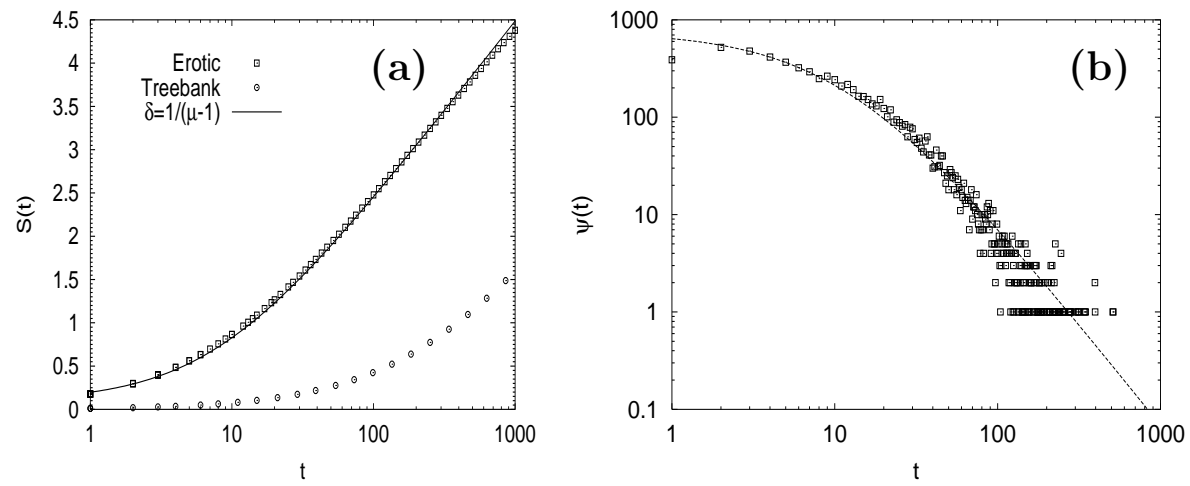

Figure 4. a) DE for salient "erotic" words for a corpus of erotic stories and offensive web pages (squares), and for the Italian reference corpus (circles). The solid line is a fit with expression $S(t)=k+\delta \ln \left(t+t_{0}\right)$, where the additional parameter $t_{0}$ is added to the original Eq. (3) to take transients into account and to improve the quality of the fit, yielding $\delta=0.91 \mathrm{~b}$ ) Non-normalized waiting time distribution for salient "erotic" words for a corpus of erotic stories. The expression for dashed line fit is $14000 \cdot(12.0+t)^{-2.1}$, yielding $\mu=2.1$.

number $c$ of classes. They found that

$$
\begin{aligned}
& p_{v}(c) \propto \frac{1}{c^{1+\eta}} \\
& p_{n}(c) \propto \frac{1}{c^{1+\eta}},
\end{aligned}
$$

where $\eta$ is a number whose absolute value is (much) smaller than 1.

On the same line, other authors 13 found a "small world" topology 14, by looking at the number of synonyms in an English thesaurus, for each English lemma. We can therefore assume that this kind of structure is general for any language. Let us therefore imagine that the paradigmatic structure of concepts is a scale-free network and consider a random walk in this "cognitive space". Let us make the following assumptions:

1) The statistical weight of the $i$-th node is $\omega_{i} \sim c_{i}$;

2) Ergodicity, and therefore that the characteristic recurrence time is $\tau_{i} \sim c_{i}^{-1}$;

3) The same form for all nodes, $\psi_{i}(t)=\left(1 / \tau_{i}\right) F\left(-t / \tau_{i}\right)$ (e.g. $\left.F(x)=\exp (-x)\right)$.

Now we imagine that selecting a concept means selecting a few neighboring nodes. This collection of nodes, due to the scale-free hypothesis, shares the same scaling properties of the complete scale free network, namely $p(c) \sim c^{-\nu}$. Therefore we have that

$$
\psi_{\text {concept }}(t)=\sum_{i} \omega_{i} \psi_{i}(t) \propto \sum_{i} c_{i}^{2} e^{-c_{i} t} \approx \int d c c^{2} e^{-c t} \frac{1}{c^{\nu}} \sim \frac{1}{t^{3-\nu}} .
$$

We recovered the intermittent model (4).

Let us now make the exercise of deriving the Zipf's law $f \propto r^{-a}$, with $a$ close to unity. Let us define a probability of frequency $P(f)$ 


$$
P(f) d f=\operatorname{prob}(r) d r \Longrightarrow P(f) \sim f^{-\frac{a+1}{a}}
$$

Next, let us notice that $P(f)$ must be a stable distribution. In fact, the Zipf's law is valid for every corpus. In particular if it is valid for corpus $A$ and for corpus $B$, it is valid also for the corpus $A+B$ where + means the concatenation of corpora. If we continue with concatenating we will have a corpus

Total Corpus $=$ Corpus A + Corpus B $+\ldots$

and we write the frequency of a word in the total corpus, $f_{\text {tot }}$ is written in terms of the single frequencies $f_{1}, f_{2}, \ldots$, and total lengths $N_{1}, N_{2}, \ldots$ of the single corpora

$$
f_{\text {tot }}=\frac{f_{1}+f_{2}+\cdots}{N_{1}+N_{2}+\cdots}=\frac{1}{\sum_{i} N_{i}} \sum_{i} f_{i}
$$

i.e., the Generalized Central Limit Theorem 15 applies. This means that the probability of frequency $P(f)$ is a Lévy $\alpha$-stable distribution. This probability of finding $f$ occurrences of a word in a corpus of a given length can be identified with $p(x ; t)$ of Section II, if we take into consideration the parameter $t$. We have earlier noticed that $p(x ; t)$ in language is Lévy process, with $\delta \sim 1$, and therefore with a tail $P(f) \sim f^{-2}$. In other words through (11) we recover (11) i.e. the Zipf's law.

\section{Conclusions}

In this paper we have shown that a cognitive process governing human language may be identified, and that it has a complexity both in the syntagmatic and in the paradigmatic axis. The scaling properties of both axes are related to each other, and are reflected by the celebrated Zipf's law. This study was conducted using Italian written corpora, but decades of studies on the generality of the Zipf's law lead us to suppose that our results are language independent, and that the language complexity that we are revealing is genuine and important. In fact, for any concept, we have a scaling index associated with an intermittent dynamical model that rests at the border between ergodicity and non-ergodicity, since the Zipf's law is theoretically consistent with $\delta=1$. Moreover, in a specialistic test we see a tendency to drift, for salient words, towards ergodicity $(\delta \approx 0.91$ in the reported experiment). This behavior can be interpreted as the balance between two opposite needs for human language, namely learnability, i.e. the possibility for a child to learn a language by examples, and variability, to explore an infinite cognitive space.

We propose as a future work to study language complexity in children during learning years, and in psychopathological subjects. We imagine, if the theory presented herein is validated by more extensive work, that the simple study of the individual Zipf's laws can provide a reasonable non-invasive diagnostic method for certain mental diseases.

From a Language Engineering point of view, this study provides a theoretical background for a completely new strategy of automatic text categorization. A prototype is being implemented as a semantic filter 10. We think that the proposed 
test for informativeness for a set of markers can also be important for many exploratory studies in time series analysis. For instance, it may become important to identify crucial semantic markers in a flow of data.

\section{References}

1. K. Silverman, The Subject of Semiotics, (Oxford Univ. Press, New York, 1983).

2. GK Zipf, Psycho-Biology of Languages (MIT Press, Cambridge MA, 1965)

3. P. Manneville, J. Physique 41, 1235 (1980).

4. P. Grigolini, L. Palatella, G. Raffaelli, Fractals 9, 439 (2001).

5. P. Allegrini, R.Balocchi, S. Chillemi, P. Grigolini, P. Hamilton, R. Maestri, L. Palatella, G. Raffaelli, Phys. Rev. E 67, 062901 (2003).

6. P. Allegrini, V. Benci, P. Grigolini, P. Hamilton, M. Ignaccolo, G. Menconi, L. Palatella, G. Raffaelli, N. Scafetta, M. Virgilio, J. Yang, Chaos, Solitons \& Fractals 15, 517 (2003).

7. S. M. Mega, P. Allegrini, P. Grigolini V. Latora, L. Palatella, A. Rapisarda, S. Vinciguerra, Phys. Rev. Lett. 90, 188501 (2003).

8. P. Allegrini, M. Barbi, P. Grigolini and B. J. West, Phys. Rev. E 52, 5281 (1995); P. Allegrini, M. Buiatti, P. Grigolini and B. J. West, Phys. Rev. E 58, 3640 (1998).

9. N. Scafetta, V. Latora, P. Grigolini, Phys. Lett. A 299, 565 (2002); N. Scafetta, V. Latora, P. Grigolini, Phys. Rev. E 66, 031906 (2002).

10. Visit URL http://www.poesia-filter.org for all information about Poesia(Public Open-source Environment for a Safer Internet Access), European Project Number IAP 2117/27572 (2002), and the open-source Poesia filter.

11. P. Allegrini, P. Grigolini, L. Palatella, G. Raffaelli, M. Virgilio, in Emergent Nature, ed M.M. Novak (World Scientific, Singapore, 2002).

12. P. Allegrini, S. Montemagni and V. Pirrelli, in COLING Proceedings, (ed. COLING, Saarbruecken 2000); P. Allegrini, S. Montemagni, V. Pirrelli, Rivista di Linguistica Computazionale, in press.

13. A.E. Motter, A.P.S. de Moura, Y.-C. Lai, and P. Dasgupta, Phys. Rev. E 9, 065102 (2002).

14. D.J.Watts and S.H.Strogatz, Nature 393, 440 (1998); A.-L.Barabási, Linked, The New Science of Networks, (Perseus Publishing, Cambridge, MA, 2002).

15. W. Feller, An introduction to probability theory and its applications, vol. 1, (Wiley, New York, 1971). 